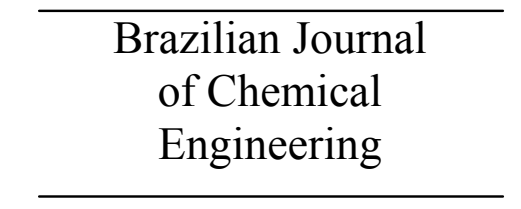

ISSN 0104-6632

Printed in Brazil

www.abeq.org.br/bjche

Vol. 26, No. 02, pp. 415 - 425, April - June, 2009

\title{
LIQUID - LIQUID EXTRACTION OF MATRINE USING TRPO/CYCLOHEXANE REVERSE MICELLES
}

\author{
Lichun Dong ${ }^{*}$, Wenping Li, Xiaohua Zhou, Qin Kang and Weifeng Shen \\ College of Chemistry and Chemical Engineering, Phone: 86-13640514654, Fax: 86-23-65111179, \\ Chongqing University, Chongqing, 400030, P.R. China. \\ E-mail: lcdong72@gmail.com
}

(Submitted: August 18, 2008 ; Revised: October 12, 2008 ; Accepted: October 13, 2008)

\begin{abstract}
Reverse micellar extraction has been widely used in the purification of biomolecules. However, reverse micelles formed by ionic surfactants can only be employed for the extraction of biomolecules that are charged in the extraction system with the electrostatic interaction between surfactants and solutes as the driving force. In this study, the extraction of matrine by using reverse micelles formed by non-ionic TRPO surfactants was studied. Theoretical analysis and experimental results demonstrated that the driving force of the extraction is the coordination forces between matrines and TRPOs. Using this coordination-based reverse micellar extraction, matrine can be efficiently separated from oxymatrine and other components in the raw matrine materials. Experimental studies showed that the factors affecting matrine extraction include $\mathrm{pH}$ value and TRPO concentration. The existence of ions in the system does not affect the partition coefficient significantly and the addition of a small amount of chloroform in the solution of reverse micelles was found to improve the extraction significantly.

Keywords: Reverse micellar extraction; Coordination interaction; TRPO reverse micelles; Matrine; Oxymatrine.
\end{abstract}

\section{INTRODUCTION}

Matrine, a kind of quinolizidine alkaloid, is reported to exhibit remarkable biomedical functions (sedative, depressant, anti-viral, anti-tumor, antibacteria, anti-inflammatory, resisting skin fibrosis, improving cardiovascular and neural functions) (Liu et al., 2007; Feng and Tang, 2005; Hoang et al., 2007). During the past decades, the production of matrine from natural plants i.e., Sophora flavescens, Sophora alopecuroides L., has attracted considerable attention from pharmaceutical researchers. All the reported methods include two steps: the first step is to extract all alkaloids from natural plants using methods such as $\mathrm{CO}_{2}$ supercritical extraction (Jian et al., 2007), macro-porous resin adsorption (Qin et al., 2007), molecularly imprinted solid-phase extraction (Lai et al., 2003), thin-layer chromatography (Ding et al., 2004) and ion-paired HPLC (Zhang et al.,
2007); the second step is to separate the alkaloids to acquire pure matrine. Jian et al. (2007) first extracted alkaloids from Sophora flavescens Ait. using supercritical fluid extraction (SFE). The crude alkaloids were then separated by high-speed countercurrent chromatography (HSCCC) to produce matrine, oxymatrine, and oxysophocarpine. In the study by Qin et al. (2007), the alkaloids were first extracted from Sophora alopecuroides L. by adsorption using macro-porous resin. The alkaloid monomers were further isolated by gradient elution with ethanol/water solutions of different volume ratios at reduced pressure. Molecularly imprinted solid-phase extraction was studied by Lai et al. (2003) to separate raw matrine from Sophora flavescens Ait; the marine of high purity was then produced by methanol/water washing and methanol/glacial acetic acid elution. All of these methods were demonstrated to be effective to

*To whom correspondence should be addressed 
produce matrine. Especially at the step to separate matrine with other alkaloids monomers (oxymatine, oxysophocarpine), however, all have their limitations such as being time-consuming, using too much organic solvents, etc. A new method is greatly needed to separate matrine from other alkaloids more efficiently and reduce the use of organic solvent at the same time.

The application of reverse micellar extraction in bio-separation has attracted considerable attention in the past two decades because the technique shows advantages in the ease of scale-up, reusable surfactant and solvent and continuous separation of biological molecules from fermentation mixtures, etc. Numerous studies have been carried out on protein, amino acid, and DNA purification using reverse micellar extraction (Wang et al., 1995; Rabie and Vera, 1996; Krieger et al., 1997; Hashimoto et al., 1998; Cardoso et al., 1999a; Jarudilokkul et al., 2000; Zhang et al., 2000; Lou et al., 2001; Liu et al., 2004; Wang et al., 2004). Among them, the reverse micellar extraction of proteins has been extensively studied and the extraction mechanism is well understood. For the extraction of proteins, reverse micelles formed by ionic surfactants were mostly employed. The transfer of proteins from aqueous phase to the reverse micelles is primarily due to the electrostatic interactions between proteins and the surfactants. Since the last decades, researchers have studied the extraction of proteins based on affinity interaction using reverse micellar solutions formed by nonionic surfactants (Guadalupe et al., 1992; Chang et al., 1997; Naoe et al., 1998; Ichikawa et al., 2000; Liu et al., 2007). By incorporating affinity ligands into the system, proteins can be selectively extracted by a biospecific affinity interaction. Because the "water pool" in reverse micelles can be adjusted, reverse micellar extraction has also been demonstrated to be a promising method to purify bio-materials with small molecules (Zhou et al., 2008, Dong et al., 2008). However, constrained by the working mechanism, reverse micelles formed by ionic surfactants can only be employed for the extraction of bio-molecules that are charged in the extraction system. In order to extract small biomolecules that are not charged, non-ionic surfactants and a novel working mechanism have to be considered.

TRPO (trialkyl phosphine oxide) is a kind of nonionic surfactant, which has been used in the extraction of metals (Xin et al., 2002) and organic acids (Wang et al., 2003) as coordination agent. In this paper, the extraction of matrine using TRPO/cyclohexane reverse micelles was studied; the driving force is believed to be the coordination forces between TRPOs and matrine, which was first rationalized by theoretical analysis and further verified by experimental result. To the best of our knowledge, we are the first group to report reverse micellar extraction with coordination as the driving force. The goal of the study is to continue the effort to extend the application of reverse micellar extraction to the purification of bio-materials with small molecules.

\section{EXPERIMENTS}

\section{Materials and Reagents}

TRPO was purchased from Shanghai Rare-earth Co. Ltd., China; Standard sample of matrine (Purity $>98 \%$ ), oxymatrine (Purity $>98 \%$ ), and raw matrine sample (produced from roots of Sophora flavescens Ait, matrine + oxymatrine $>60 \%$ ) were form Xi' an Honson Biotechnology Co. Ltd., Xi'an, China.

\section{Experimental Methods and Procedures}

\section{a) Preparation of TRPO Reverse Micelles}

TRPO was first dehydrated using $4 \AA$ zeolite for $48 \mathrm{~h}$, mixed with cyclohexane in an iodine flask, and then a suitable amount of D.I. water was added. The expected reverse micellar solution was finally obtained after thoroughly stirring for several hours. The solution should be transparent and does not adsorb UV and visible light. The $\mathrm{W}_{0}\left(\mathrm{~W}_{0}=\right.$ moles of water /moles of TRPO) of TPRO/cyclohexane reverse micelles prepared in this study varies from 15 to 3 and the estimated "pool" radius from $10 \mathrm{~nm}$ to $2 \mathrm{~nm}$ (Cardoso et al., 1999b; Reng et al., 2004).

To confirm the formation of reverse micelles, the IR spectra of TRPO (before dehydrating), dehydrated TRPO/cyclohexane solution, and TRPO/cyclohexane reverse micelles was recorded using a MAGMA - IR550 spectrometer (Nicolet Company, USA).

\section{b) Preparation of Solutions of Matrine, Oxymatrine and Raw Matrine}

The solutions of matrine (or oxymatrine) were prepared by dissolving standard matrine (or oxymatrine) using D.I. water in an iodine flask followed by filtering using a microporous membrane with pore size of $0.45 \mu \mathrm{m}$.

The same procedure was followed to prepare the raw solution. The concentration of matrine and oxymatrine in the raw solution was measured using capillary electrophoresis. All the prepared solutions were stored at $4^{\circ} \mathrm{C}$ before being used. 


\section{c) Extraction and Strip-Extraction Experiments}

The solution of TRPO/cyclohaxane reverse micelles of known volume was first put into an iodine flask, then the solution of matrine (or oxymatrine, raw matrine) was added for extraction for $45 \mathrm{~min}$ with a stirring speed of $200 \mathrm{rpm}$. The extraction was processed at $30{ }^{\circ} \mathrm{C}$ and a controlled $\mathrm{pH}$ value. The mixture was allowed to stand for 2 min to separate the extract and raffinate phase before the extract and raffinate phases were gathered, respectively. The quantity of matrine in the raffinate phase was measured and the extraction efficiency and partition coefficient were calculated according to Eq. 1 and Eq. 2, respectively.

Extraction Efficiency $(\%)=$

Quantity of matrine in extract phase $\times 100 \%$

Total quantity of matrine

Partititon Coefficient (D) =

Concentration of matrine in extract phase

Concentration of matrine in raffinate phase

The extract phase was then transferred into another iodine flask, then strip-extraction liquid (DI water with the $\mathrm{pH}$ value adjusted using $\mathrm{NaOH}$ ) was added for strip-extraction for $40 \mathrm{~min}$. As in the extraction, the strip-extraction was processed at $30{ }^{\circ} \mathrm{C}$ and with a stirring speed of $200 \mathrm{rpm}$. After the mixture was allowed to stand for $2 \mathrm{~min}$ to separate the extract and raffinate phase, the quantity of matrine in the extract phase was measured. The strip-extraction efficiency was calculated according to Eq. 3 .

Strip-extraction Efficiency $(\%)=$

Quantity of matrine in strip-extraction extract phase $\times 100 \%$

Total quantity of matrine in reverse micellar solution

\section{d) Quantitative Measurement of Matrine and Oxymatrine}

The quantity of matrine (oxtmatrine) was measured using a method of capillary electrophoresis (Yao et al., 1996, Na et al., 2003). The buffer used in capillary electrophoresis is $25 \mathrm{mmol} / \mathrm{L}$ borax solution, which contains $30 \mathrm{mmol} / \mathrm{L}$ SDS and $35 \%$ ethanol (by volume). The sample for electrophoresis is injected at $25 \mathrm{KV}$ and the sample for electromigration is injected at $25 \mathrm{KV} \times 4 \mathrm{sec}$; the wavelength of testing is $208 \mathrm{~nm}$. The quantity of all contents was determined by the internal standard method with ephedrine as the internal standard sample. The calculation is by Eq. 4 .

$$
C_{X}=f A_{X} \frac{C_{S}^{\prime}}{A_{S}^{\prime}}
$$

where $\mathrm{CX}$ and $\mathrm{AX}$ are the concentration and the peak area of the sample, respectively. $C_{S}^{\prime}$ and $A_{S}^{\prime}$ are the concentration and peak area of the internal standard sample, respectively; $\mathrm{f}$ is the correction factor.

\section{THEORETICAL ANALYSIS OF MATRINE EXTRACTION USING TRPO REVERSE MICELLES}

Neither matrine nor oxymatrine are soluble in cyclohexane or anhydrous TRPO (Sun and Xie, 2003), while matrine can be dissolved in the solution of TRPO/cyclohexane reverse micelles. The only places that the solution of TRPO/cyclohexane reverse micelles can dissolve matrines are the "water pools". The extraction of materine by the solution of $\mathrm{TRPO} /$ cyclohexane is, therefore, a kind of reverse micellar extraction.

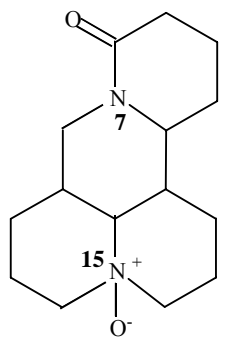

(b)

Figure 1: Molecular Structures of Matrine (a) and Oxymatrine (b). 


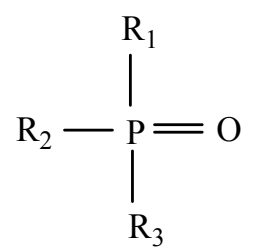

Figure 2: Molecular Structure of TRPO. $\mathrm{R}_{1}, \mathrm{R}_{2}, \mathrm{R}_{3}=\mathrm{CH}_{3}\left(\mathrm{CH}_{2}\right)_{7}$ or $\mathrm{CH}_{3}\left(\mathrm{CH}_{2}\right)_{5}$.

The molecular structure of both matrine and oxymatrine is planar (Fig. 1). The difference between the molecular structure of matrine and that of oxymatrine is that the $\mathrm{N}^{15}$ atom in oxymatrine is coordinated to an oxygen atom. Both $\mathrm{N}^{15}$ and $\mathrm{N}^{7}$ in matrine molecules are tertiary nitrogens and have two lone-pair electrons. However, $\mathrm{N}^{7}$ is adjacent to a carbonyl group; the electrons of $\mathrm{N}^{7}$ conjugate with the carbonyl due to the strong electron-attracting capability of the carbonyl group, with the result that $\mathrm{N}^{7}$ cannot share the lone-pair electrons and form coordination bonds with atoms having empty orbitals. Although connected to three $\mathrm{C}$ atoms, $\mathrm{N}^{15}$ in matrine can easily form coordination bonds with atoms having empty orbitals via the lone-pair electrons because $\mathrm{N}$ is more electronegative than $\mathrm{C}$.

TRPO is a kind of nonionic surfactant (Fig. 2); the oxygen atom in TRPO has an empty orbital that can form a coordination bond with atoms with lonepair electrons like the $\mathrm{N}^{15}$ atom in matrine. Because TRPO is a kind of non-ionic surfactant, the driving force for TRPO/cyclohexane reverse micellar extraction of matrine is surely not the electrostatic force between the TRPOs and matrines. According to the molecular structures of matrine and TRPO, the driving force for the extraction is believed to be the coordination force between them. When matrine meets TRPO in the reverse micellar system, matrine can form a TRPO-matrine complex with TRPO via a $\mathrm{N}^{15}-\mathrm{O}$ coordination bond and be extracted into TRPO reverse micelles. The $\mathrm{N}^{15}$ in oxymatrine is already coordinated with an oxygen atom (Fig. 1), thus cannot coordinate with TRPO. Therefore, oxymatrine can be separated from matrine by using TRPO reverse micellar extraction.

According to the extraction mechanism, the followed reaction occurs during the extraction of matrine using TRPO reverse micelles.

matrine $-\mathrm{N}^{15}+\mathrm{O}=\mathrm{P}-\mathrm{R}_{3} \stackrel{\mathrm{K}_{\mathrm{eq}}}{\longleftrightarrow}$

matrine $-\mathrm{N}^{15} \rightarrow \mathrm{O}=\mathrm{P}-\mathrm{R}_{3}$

where $\mathrm{K}_{\mathrm{eq}}$ is the reactive equilibrium constant. When the extraction reaches equilibrium

$$
\mathrm{K}_{\mathrm{eq}}=\frac{\left[\text { matrine }-\mathrm{N}^{15} \rightarrow \mathrm{O}=\mathrm{P}-\mathrm{R}_{3}\right]}{\left[\text { matrine }-\mathrm{N}^{15}\right]\left[\mathrm{O}=\mathrm{P}-\mathrm{R}_{3}\right]}
$$

In aqueous solution, the $\mathrm{N}^{15}$ atom of matrine can bond with a $\mathrm{H}^{+}$ion to become quaternary, as in Eq. 7. The $\mathrm{N}^{15}$ is then positively charged and can further bond with negatively charged ions in the extraction system, which causes matrine to lose its coordination ability (Yang and Ma, 1997).

$$
\text { Matrine }-\mathrm{N}^{15}+\mathrm{H}^{+} \stackrel{\mathrm{K}_{1}}{\longleftrightarrow} \text { Matrine }-\mathrm{N}^{15} \mathrm{H}^{+}
$$

where $K_{1}$ is the equilibrium constant of the reaction. When the reaction reaches equilibrium

$$
\mathrm{K}_{1}=\frac{\left[\text { Matrine }-\mathrm{N}^{15} \mathrm{H}^{+}\right]}{\left[\text {Matrine }-\mathrm{N}^{15}\right]\left[\mathrm{H}^{+}\right]}
$$

By modifying Eq. 2, the partition coefficient of matrine can be calculated from Eq. 9 .

$\mathrm{D}=\frac{\text { Quantity of matrines in extract phase }}{\mathrm{R} \times \text { Quantity of matrines in raffinate phase }}$

where $\mathrm{R}$ is the phase volume ratio between organic phase (solution of TRPO reverse micelles) and aqueous phase. The quantity of matrines extracted into the extract phase is equal to the quantity of Matrine $-\mathrm{N}^{15} \rightarrow$ TRPO in the extraction system, the quantity of matrines left in the raffinate phase is equal to the sum of the quantity of matrines that do not form complexes with TRPOs and the quantity of protonated matrines with $\mathrm{N}^{15}$ as quaternary nitrogens. Thus Eq. 9 can be transformed to Eq. 10.

$$
\mathrm{D}=\frac{\left[\text { Matrine }-\mathrm{N}^{15} \rightarrow \mathrm{O}=\mathrm{P}-\mathrm{R}_{3}\right]}{\mathrm{R}\left(\left[\text { Matrine }-\mathrm{N}^{15}\right]+\left[\text { Matrine }-\mathrm{N}^{15} \mathrm{H}^{+}\right]\right)}
$$

Substituting Eq. 6 and Eq. 8 into Eq. 10, we get Eq. 11: 


$$
\begin{aligned}
\mathrm{D}= & \frac{\mathrm{K}_{\mathrm{eq}}\left[\text { Matrine }-\mathrm{N}^{15}\right]\left[\mathrm{O}=\mathrm{P}-\mathrm{R}_{3}\right]}{\mathrm{R}\left(\left[\text { Matrine }-\mathrm{N}^{15}\right]+\mathrm{K}_{1}\left[\text { Matrine }-\mathrm{N}^{15}\right]\left[\mathrm{H}^{+}\right]\right)}= \\
& \frac{\mathrm{K}_{\mathrm{eq}}\left[\mathrm{O}=\mathrm{P}-\mathrm{R}_{3}\right]}{\mathrm{R}\left(1+\mathrm{K}_{1}\left[\mathrm{H}^{+}\right]\right)}
\end{aligned}
$$

In Eq. $11, \mathrm{~K}_{\mathrm{eq}}$ and $\mathrm{K}_{1}$ are all constants. Eq. 11 shows that the partition coefficient increases with an increase of the concentration of TRPO, and decreases with an increase of the concentration of $\mathrm{H}^{+}$. In the following sections of the paper, the experimental results of the extraction of matrine using TRPO reverse micelles are discussed to verify the theoretical analysis.

\section{EXPERIMENTAL RESULTS AND DISCUSSION}

\section{IR Analysis of the Formation of Reverse Micelles}

The IR spectra of TRPO, TRPO/cyclohexane solution dehydrated using $4 \AA$ zeolite, and TRPO/cyclohexane reverse micellar solution are shown in Fig. 3. Since TRPO of industrial grade contains a small amount of water, its IR spectrum has two peaks of $-\mathrm{OH}$ at $1643 \mathrm{~cm}^{-1}$ (associated state) and $3391 \mathrm{~cm}^{-1}$ (free state), respectively. There is also a strong absorbance at $1158 \mathrm{~cm}^{-1}$ corresponding to the $\mathrm{P}=\mathrm{O}$ group in the spectrum. After being dehydrated using $4 \AA$ zeolite, the peaks corresponding to $-\mathrm{OH}$ disappear in the IR spectrum of TRPO/cyclohexane solution. Moreover, the strong absorbance of $\mathrm{P}=\mathrm{O}$ weakens and moves to a lower wave number. The reason for this change is that the hydrophobic alkyl groups of TRPO are associated with cyclohexane, the polar $\mathrm{P}=\mathrm{O}$ groups aggregate to form hydrophilic "cores", the characteristic absorbance of $\mathrm{P}=\mathrm{O}$ is, therefore, screened. After D.I. water was added to TRPO/cyclohexane solution, an absorbance at $1659 \mathrm{~cm}^{-1}$ corresponding to $-\mathrm{OH}$ in the associated state appears in the IR spectrum of the system, whereas the absorbance corresponding to $\mathrm{OH}$ in the free state is still absent. The rational reason for this result is that the added water is confined in the "pool" formed by TRPO reverse micelles; the binding with $\mathrm{P}=\mathrm{O}$ makes the properties of water in the "pools" different from the free water. Therefore, it can be concluded that the transparent water-containing TRPO/cyclohexane system is a solution of TRPO reverse micelles.

\section{Factors Affecting Matrine Extraction}

Theoretical analysis indicated that the matrine extraction is affected by $\mathrm{pH}$ and TRPO concentration. In order to verify the theoretical prediction, a series of experiments were performed to study the possible factors affecting the extraction, including $\mathrm{pH}$, TRPO concentration, ions, etc.

\section{a) Effect of pH on Matrine Extraction}

Fig. 4 shows the experimental partition coefficient versus $\mathrm{pH}$ of the matrine solution. The experiments were set up to extract matrine from standard matrine solutions previously prepared. The partition coefficient increases with an increase of $\mathrm{pH}$ value from 4 to 9 . As shown in Eq. 7, the $\mathrm{N}^{15}$ in matrine molecules can be a tertiary amine or a quaternary amine, whose concentration ratio is dependent on the $\mathrm{pH}$ of the system. With an increase of $\mathrm{pH}$, the decrease of $\mathrm{H}^{+}$concentration increases the concentration of tertiary amines in the extraction system, which is in favor of the formation of matrine $-\mathrm{N}^{15} \rightarrow \mathrm{O}=\mathrm{P}=\mathrm{R}_{3}$ complexes. Therefore, the experimental partition coefficient increases with an increase of the $\mathrm{pH}$ value, as predicted by Eq. 11 . When the $\mathrm{pH}$ value exceeds 9, the increase of interfacial activity of matrine could cause the emulsification of the extraction system, which would make the separation of extract phase and reaffinate phase very difficult. Therefore, the optimum $\mathrm{pH}$ value for the extraction of matrine using TRPO reverse micelles is between 8 and 9 .

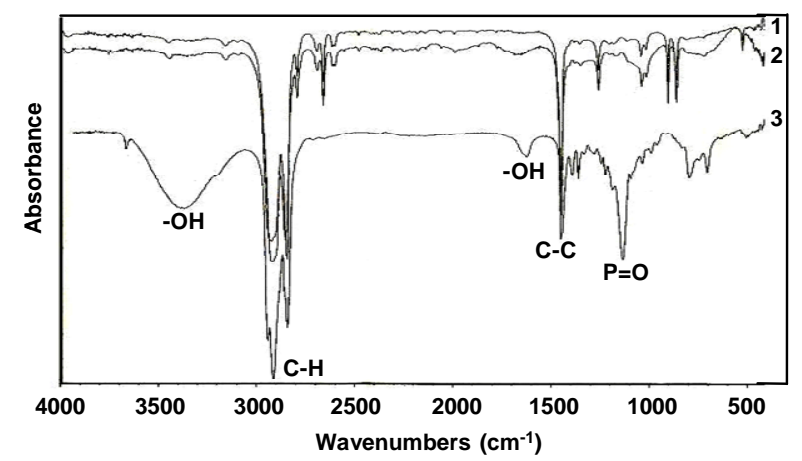

Figure 3: IR spectra of dehydrated TRPO/cyclohexane solution (1), TRPO/ cyclohexane reverse micelle (2), and TRPO (3). 


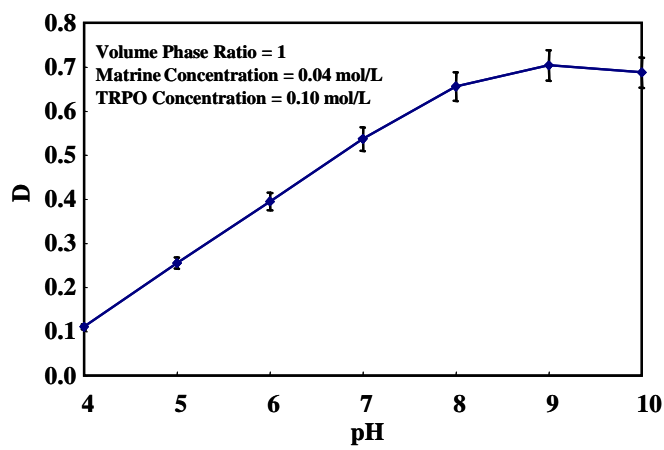

Figure 4: Experimental partition coefficient versus the $\mathrm{pH}$ value of the matrine solution.

\section{b) Effect of TRPO Concentration on Matrine Extraction}

Fig. 5 shows the experimental partition coefficient versus TRPO concentration. As in the previous section, the experiments were performed to extract matrine from standard matrine solutions prepared. When the concentration of TRPO is in the range of 0.05 to $0.25 \mathrm{~mol} / \mathrm{L}$, the partition coefficient increases with an increase in TRPO concentration, which agrees well with the theoretical prediction of Eq. 11. The partition coefficient reaches a plateau value when the TRPO concentration is higher than $0.25 \mathrm{~mol} / \mathrm{L}$. The water in the "water pools" of reverse micelles can be categorized into two classes: the "free" water and the "bound" water that is bounded to the surfactants (Menger and Saito, 1978; Jolivalt et al., 1989; Ono et al., 1996). The properties of the "bound" water are different from those of the "free" water and the extraction capability of the reverse micelles is mostly dependent on the total amount of "bound" water. As the concentration of the TRPO increases, the "pool" size of the reverse micelles decreases. A larger portion of the water inside the reverse micelles is bound to the surfactants and becomes "bound" water, which benefits the extraction and increases the partition coefficient. However, with the amount of "free" water decreasing, the availability of "free" water becomes

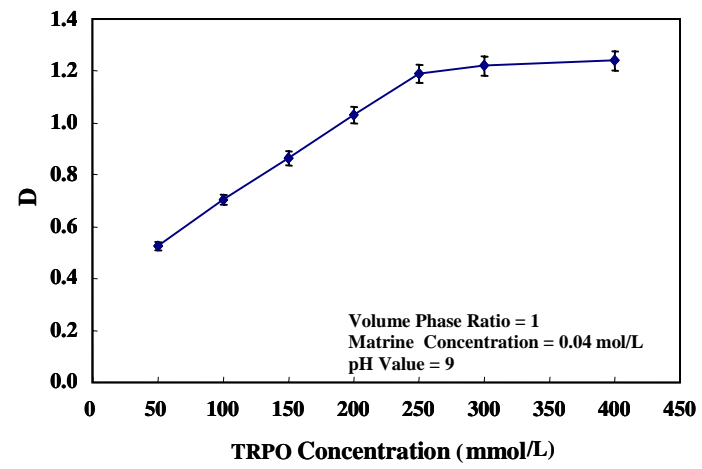

Figure 5: Experimental partition coefficient versus TRPO concentration. more and more difficult with the increase of TRPO concentration. When all the water inside the reverse micelles becomes "bound" water, the increase of the concentration of the TRPO reverse micelles does not affect the extraction any further.

\section{c) Effect of Ions on Matrine Extraction}

In the extraction using ionic reverse micelles with electrostatic interactions as the driving force, the existence of ions in the system always affects the extraction significantly because ions can influence the electrostatic interaction between surfactants and solutes in several ways: salting out effect, screening effect, etc (Huang and $\mathrm{Gu}, 1996$; Zhou et al., 2008). However, in this study, TRPO is a kind of non-ionic surfactant and the driving force of the extraction is the coordination forces. Thus, the existence of ions in the system should not affect the extraction significantly. To verify this hypothesis, the partition coefficient of matrine was studied with $\mathrm{CaCl}_{2}$ present in the system was studied and the results are shown in Fig. 6. The figure shows that the partition coefficient hardly changes at different $\mathrm{CaCl}_{2}$ concentrations. This observation confirmed that the existence of ions in the system does not affect the coordination interaction between TRPOs and matrines or the partition coefficient significantly.

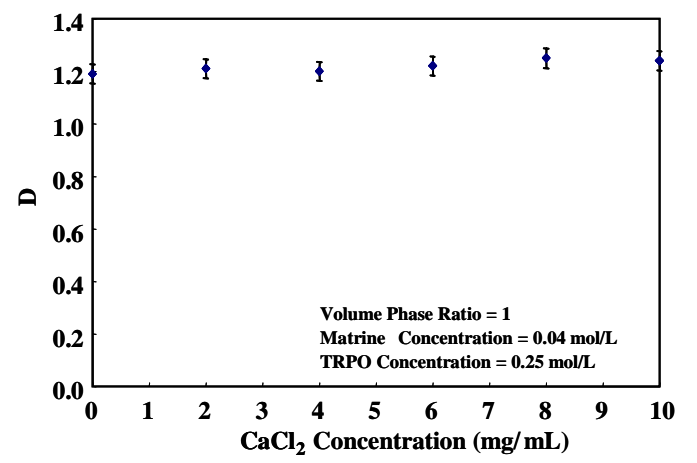

Figure 6: Experimental partition coefficient versus $\mathrm{CaCl}_{2}$ concentration. 


\section{d) Effect of Chloroform on Matrine Extraction}

In the study, the addition of a small amount of chloroform has been found to increase the partition coefficient remarkably. Fig. 7 shows that the partition coefficient of matrine increases from 1.2 to 3.7 when the weight concentration of chloroform in the solution of TRPO reverse micelles increases from 0 to $6.0 \%$. The exact working mechanism of chloroform is not clear yet and requires a more detailed study. According to previous studies (Liu et al., 2007), a possible explanation is that chloroform, working as co-solvent, can help surfactants to dissolve better in the organic solvent, adjust the interaction between the polar heads of the surfactants, and make the reverse micelles have a suitable "pool" size for extraction.

\section{e) Effect of Extraction Time on Matrine Extraction}

Fig. 8 shows the partition coefficient of matrine versus extraction time. It takes about 45 min for the extraction to reach equilibrium and the partition coefficient then reaches a plateau after $45 \mathrm{~min}$.

\section{The Strip-Extraction of Matrine}

Using D.I. water of different $\mathrm{pH}$ values as strip-extraction liquid, the strip-extraction of matrine from the extract phase (the TRPO reverse micelles with matrine) obtained from the extraction experiments was studied. Opposite to the extraction, the strip-extraction efficiency decreases with an increase in $\mathrm{pH}$. With an increase of $\mathrm{pH}$ value, Eq. 7 moves to the left, the concentration of matrines with $\mathrm{N}^{15}$ as tertiary amines increases, strengthening the coordination interaction between matrines and TRPOs, and the strip-extraction efficiency decreases. Fig. 9 shows that the single strip-extraction efficiency decreases from $67 \%$ to $15 \%$ when the $\mathrm{pH}$ value of the strip-extraction increases from 3 to 10 , which agrees well with the theoretical prediction.

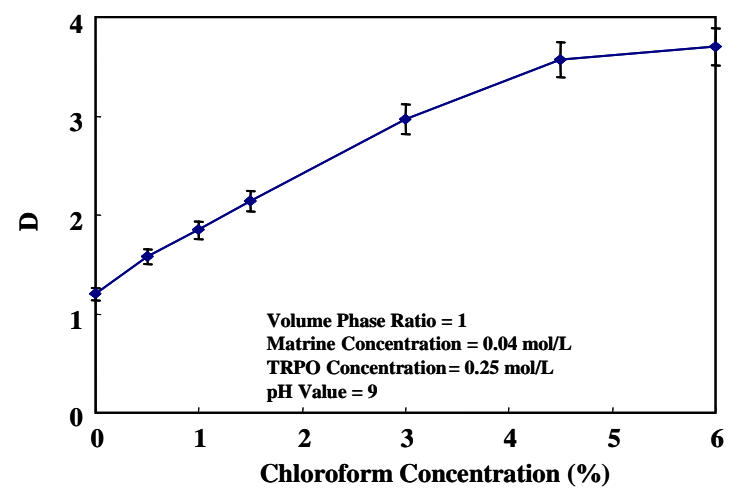

Figure 7: Experimental partition coefficient versus chloroform concentration.

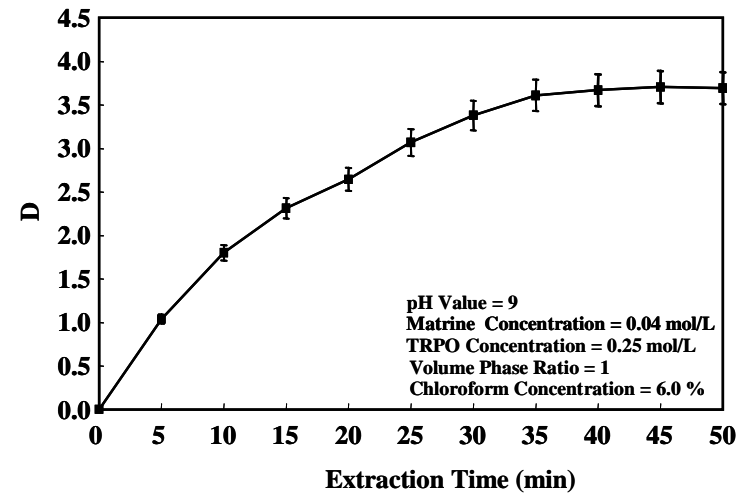

Figure 8: Experimental partition coefficient versus chloroform concentration.

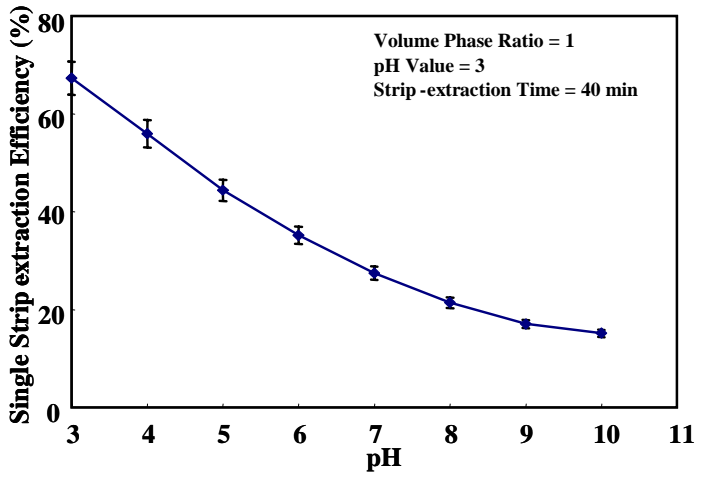

Figure 9: Experimental strip-extraction coefficient versus $\mathrm{pH}$ value. 
Experimental Study of Oxymatrine Extraction Using TRPO Reverse Micelles

Theoretical analysis indicated that oxymatrine can not be extracted by TRPO reverse micelles since it can not coordinate with TRPO. To verify this prediction, extraction of oxymatrine from standard oxymatrine solution was studied. Fig. 10a shows the experimental extraction efficiency of oxymatrine at different chloroform concentrations. Although the addition of chloroform increases the extraction of oxymatrine, like that of matrine, the extraction efficiency of oxymatrine is much lower, which confirms the theoretical analysis. Fig. 10b shows the separation factor between matrine and oxymatrine at different chloroform concentrations, where the separation factor is the ratio between extraction efficiency of matrine and that of oxymatrine at the same operating condition. The separation factor is 16.5 with no chloroform. With chloroform added, the separation factor first decreases with an increase in chloroform concentration and reaches a minimum, then increases with a further increase of chloroform concentration. The separation factor is about 15.5 when the chloroform concentration is $6 \mathrm{wt} \%$. Therefore, the matrine extraction should be operated at higher chloroform concentration because the extraction has higher matrine extraction efficiency as well as a better separation factor.

\section{The Separation of Matrine with Oxymatrine}

On the basis of single-factor experiments previously discussed, the optimum conditions for

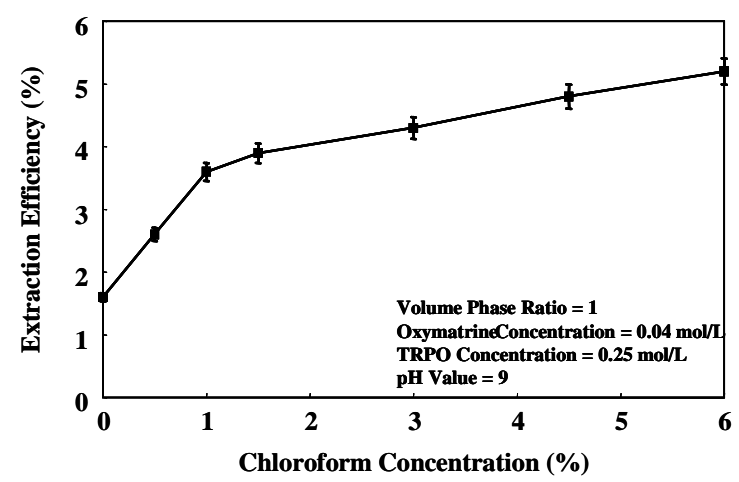

(a) matrine extraction and strip-extraction from raw matrine solution were investigated via two groups of orthogonal experiments. The optimum operating conditions for extraction are: $\mathrm{pH}=9.0$, TRPO concentration $=0.25 \mathrm{~mol} / \mathrm{L}$, chloroform concentration $=6 \mathrm{wt} \%$, phase ratio $=1: 1(\mathrm{~V} / \mathrm{V})$, extraction time $=45 \mathrm{~min}$. The optimum operating conditions for strip-extraction are: $\mathrm{pH}=3$, phase ratio $=1: 1(\mathrm{~V} / \mathrm{V})$, and strip-extraction time $=$ $40 \mathrm{~min}$.

With matrine and oxymatrine concentration in the raw matrine solution of $0.047 \mathrm{~mol} / \mathrm{L}$ and 0.083 $\mathrm{mol} / \mathrm{L}$, respectively, the yield of the matrine can reach $60 \%$. After strip-extraction, the strip-extraction extract phase was concentrated and lyophilized. The powder of the products after lyophilization was analyzed by using capillary electrophoresis as described in the experimental section. Fig. 11 shows the results of the products after three stripextractions. The composition of the final product is: $90.2 \%$ matrine, $5.4 \%$ oxymatrine, $4.4 \%$ water and others. By the second extraction, matrine with higher purity can be produced.

Except matrine and oxymatrine, the solution of raw matrine also contains saccharide, short peptides, a small amount of oxysophocarpine and amino acids, etc. It can be seen that TRPO reverse micelle extraction can separate matrine from most of oxymatrine and all of the other components. Compared with reverse micellar extraction based on electrostatic force, this coordination interactionbased reverse micellar extraction has better selectivity because of the specific interaction between surfactant and the solvent.

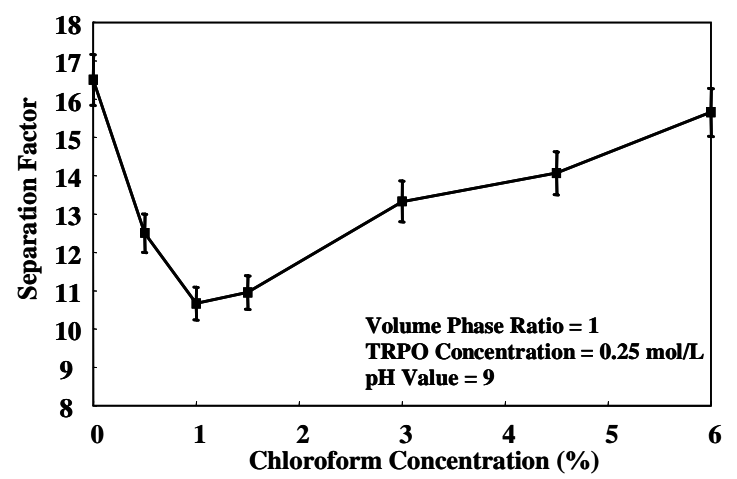

(b)

Figure 10: Experimental extraction efficiency of oxymatrine (a) and the separation factor between matrine and oxymatrine (b) versus chloroform concentration. 


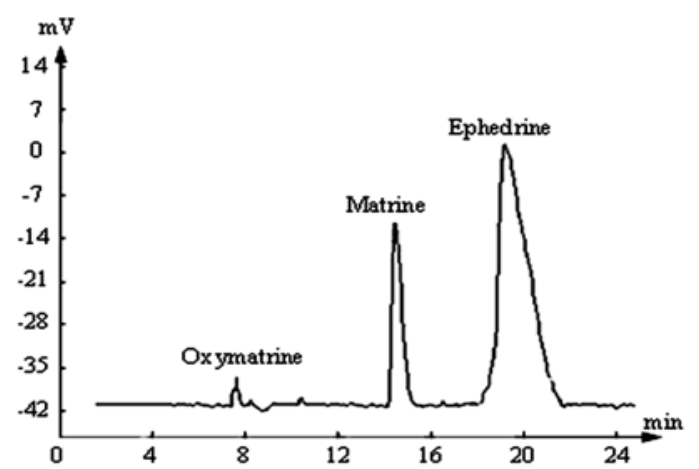

Figure 11: The capillary electrophoresis results for the final products after three strip-extractions.

\section{CONCLUSIONS}

In this paper, matrine extraction using TRPO reverse micelles was studied and the working mechanism of the extraction is verified to be the coordination forces between matrines and TRPOs. Using this coordination-based reverse micellar extraction, matrine can be efficiently separated from oxymatrine and other components in the raw matrine materials. The yield can reach $60 \%$, and the purity of matrine is more than $90 \%$. The significance of this study is that it sheds considerable light on the purification of biomaterials with small molecules by extraction using reverse micelles formed by nonionic surfactants.

The main factors affecting matrine extraction using TRPO reverse micelles include $\mathrm{pH}$ and TRPO concentration. The partition coefficient of matrine increases with an increase in $\mathrm{pH}$ of the aqueous phase and TRPO concentration when it is below $0.25 \mathrm{~mol} / \mathrm{L}$. The existence of ions in the system does not affect the extraction significantly. Moreover, the addition of a small amount of chloroform has been found to increase the partition coefficient remarkably.

\section{NOMENCLATURE}

\section{Abbrevation}

TRPO Trialkyl phosphine oxide

\section{Symbols}

$\mathrm{W}_{0} \quad$ the ratio between moles of water and that of TRPO in reverse micellar solution

D partition coefficient of extraction

$\mathrm{C}_{\mathrm{X}}$ the concentration of the sample in the capillary

electrophoresis

measurement

$A_{X} \quad$ the peak area of the sample

$(-)$

in the capillary

electrophoresis

measurement

$\mathrm{C}_{\mathrm{S}}^{\prime} \quad$ the concentration of the $\mathrm{kg} / \mathrm{mL}$ internal standard sample in the capillary electrophoresis measurement

$\mathrm{A}_{\mathrm{S}}^{\prime} \quad$ the peak area of the internal standard sample in the capillary electrophoresis measurement

$\mathrm{K}_{\mathrm{eq}} \quad$ reactive equilibrium constant

$\mathrm{K}_{1} \quad$ reactive equilibrium constant

$\mathrm{R}$ phase volume ratio for extraction

\section{ACKNOWLEDGMENTS}

The authors would like to thank the Chongqing Hanfan Company for their financial support.

\section{REFERENCE:}

Cardoso, M. M., Viegas, R. M. C., and Crespo, J. P. S. G., Extraction and re-extraction of phenylalanine by cationic reverse micelles in hollow fibre contactors, J Memb. Sci., 156 (2), 303 (1999).

Cardoso, M. M, Barradas, M. J., Kroner, K. H., and Crespo J. G., Amino acid solubilization in cationic reverse micelles: factors affecting amino acid and water transfer. J Chem. Technol. Biotechnol., 74, 801 (1999). 
Chang, Q., Chen, J., Zhang, X., and Zhao, N., Effect of the cosolvent type on the extraction of $\alpha$ amylase with reversed micelles: circular dichroism study, Enzyme. Microb. Tech., 20, 87 (1997).

Ding, P., Jiang, S., Qiao, C., and Chen, D., Isolation and purification of matrine and oxymatrine and TLC identification of Radix Sophorae Tonkinensis, Chinese Pharmaceutical Journal., 21, 333 (2004). (in Chinese)

Dong, L., Zhou, X., and Li, D., Liquid-liquid extraction of hyperoside using CTAB reverse micelles, Submitted to Can. J Chem. Eng., (2008). (accepted)

Feng, X., and Tang, H. A., Review of the pharmacological research on matrine, Chinese Journal of Pharmacoepidemiology, 6, $331-333$. (2005). (in Chinese)

Guadalupe, A. A., Sanjay, K., Eric, J. B., and Alan, J. R., Protein extraction and activity in reverse micelles of a non-ionic detergent, Biotechnol. Bioeng., 39, 806 (1992).

Hashimoto, Y., Ono, T., Goto, M., and Hatton, T. A., Protein refolding by reverse micelles utilizing solid-liquid extraction technique, Biotechnol. Bioeng., 57 (5), 620 (1998).

Hoang, B. X., Shaw, D. G., Levine, S., and Hoang C., New approach in asthma treatment using excitatory modulator. Phytother, Res., 21 (6), 554 (2007).

Huang, W., and Gu, X., Solubilization of aqueous solution of electrolytes by dodecylammonium propionate in carbon tetrachloride, Acta PhysicoChimica Sinica. 12 (1), 49 1996). (in Chinese)

Ichikawa, I., Sugiura, S., Nakajima, M., Sano, Y., Seki, M., and Furusaki, S., Formation of biocompatible reverse micellar systems using phospholipids, Biochem. Eng. J., 6, 193 (2000).

Jarudilokkul, S., Paulsen, E., and Stuckey, D. C., Lysozyme extraction from egg white using reverse micelles in a Graesser contactor: Mass transfer characterization, Biotechnol. Bioeng., 69 (4), 618 (2000).

Jian, Y., Guo, Y., Zhao, J., and Chang, K., Supercritical fluid extraction of quinolizidine alkaloids from Sophora flavescens Ait. and purification by high-speed counter-current chromatography, J Chromatogr. A, 1145 (1-2), 123 (2007).

Jolivalt, C., Minier, M., and Renon, H., Protein separation using affinity-based reverse micelles, Fluid Phase Equilib., 53 (2), 483 (1989).

Krieger, N., Taipa, M. A., Aires-barros, M. R., Eduardo, H. M. M., Jorge, L. L. F., and Joaquim,
M. S. C., Purification of the penicillium citrinum lipase using AOT reversed micelles, J Chem. Technol. Biotechnol. 69 (1), 77 (1997).

Lai, J., He, X., Jiang, Y., and Chen, F., Preparative separation and determination of matrine from the Chinese medicinal plant Sophora flavescens Ait. by molecularly imprinted solid-phase extraction, Anal. Bioanal. Chem., 375 (2), 264 (2003).

Liu, J., Xing, J., Shen, R., and Yang, C., Reverse micelles extraction of nattokinase from fermentation broth, Biochem. Eng. J., 21 (3), 273 (2004).

Liu, J., Hu, J., Zhu, Q., Li, F., Wang, J., and Sun, H., Effect of matrine on the expression of substance $\mathrm{P}$ receptor and inflammatory cytokines production in human skin keratinocytes and fibroblasts, Int. Immunopharmacol., 7 (6), 816 (2007).

Liu, Y., Dong, X., and Sun, Y., Protein separation by affinity extraction with reverse micelles of Span 85 modified with Cibacron Blue F3G-A, Sep. Purif. Technol., 53 (3), 289 (2007).

Lou, W., Zong, M., Liu, S., and Li, H., Recent progress in reverse micellar extraction of enzyme-protein, Industrial Microbiology, 31 (4), 54 (2001). (in Chinese)

Menger, F. M., and Saito, G., Adsorption, displacement, and ionization in water pools, J Am. Chem. Soc., 100, 4376 (1978).

Na, P., Mao, X., Chen, P., He, T., Gao, J., and Hou, J., Determination of Matrine and Oxymatrine in Radix Sophorae Tonkinensis by Micellar Capillary Electrophoresis, Journal of Instrumental Analysis, 22 (2), 63 (2003). (in Chinese)

Naoe, K., Ura, O., Hattori, M., Kawagoe, M., and Imari, M., Protein extraction using non-ionic reverse micelles of Span 60, Biochem Eng. J., 2, 113 (1998).

Ono, T., Goto, M., Nakashio, F., and. Hatton, T. A., Extraction behavior of hemoglobin using reverse micelles by dioleyl phosphoric acid, Biotechnol. Prog., 12 (6), 793 (1996).

Qin, X., Yuan, Y., Wu, J., and Yang, J., Separation of Alkaloids from Sophora alopecuroides L. by Adsorption Using Macroporous Resins, J Chem. Eng. Jap., 40 (2), 93 (2007).

Rabie, H. R., and Vera, J. H., Extraction of zwitterionic amino acids with reverse micelles in the presence of difference ions, Ind. Eng. Chem. Res., 35, 3665 (1996).

Reng, W., Li, S., and Wang, Y., Synthetic mechanism of hydroxyapatite nanoparticles via AOT inverse microemusions, Chin. J Mater. Res., 18 (3), 257 (2004). (in Chinese) 
Sun, W., and Xie, S., The quantitative analysis of ingredients in natural pharmaceuticals, China Press of Pharmaceutical Technology, Beijing, China, (2003). (in Chinese)

Wang, W. H., Weber, M. E., and Vera, J. H., Reverse micellar extraction of amino acids using dioctyldimethylammonium chloride, Ind. Eng. Chem. Res., 34, 599 (1995).

Wang, Y., Li, Y., Li, Y. Q., and Dai, Q., LiquidLiquid Extraction of Carboxylic Acid with Trialkyl Phosphine Oxide, Chin. Chem. Eng., 31 (6), 8 (2003).

Wang, Y., Shi, C., Gan, Q., and Dai, Y., Separation of amino acids by polymeric reverse micelle extraction, Sep. and Purif. Technol., 35 (1), 1 (2004).

Xin, R., Li, L., Song, C., and Liang, J., The extraction of rare earths by TRPO, Chin, Rare Earths, 23(4), 23 (2002). (in Chinese)

Yang, J., and Ma, J., Study on reactive extraction of cefalosporin C II, Extraction conditions, Chinese
Journal of Antibiotics, 22 (4), 258 (1997). (in Chinese)

Yao, Y., The quantitative measurement of biological alkali in plant pesicides, Inner Mongolia Petrochemical Industry, 2 (22), 31 (1996). (in Chinese)

Zhang, L., Xu, L., Tan, X., Liao, Q., Guo, W., Chen, $\mathrm{X}$, and $\mathrm{Bi}, \mathrm{K}$., Simultaneous determination of baicalin, baicalein, wogonin, oxysophocarpine, oxymatrine and matrine in the Chinese herbal preparation of Sanwu-Huangqin-Tang by ionpaired HPLC, Chromatographia, 66 (1-2), 115 (2007). (in Chinese)

Zhang, T., Liu, H., and Chen, J., Affinity Extraction of BSA by Mixed reverse micellar system with unbound triazine dye. J Chem. Technol. Biotechnol., 75 (9), 798 (2000).

Zhou, X., Dong, L., and Li, D., A Comprehensive study of Extraction of hyperoside from Hypericum perforatum L. using CTAB reverse micelles, J Chem. Technol. Biotechnol. 83, 1413 (2008). 